\title{
1 Computational Tool for Ensemble Averaging of Single-Molecule Data
}

2
Thomas Blackwell1, W. Tom Stump1, Sarah R. Clippinger, Michael J. Greenberg1,*

1Department of Biochemistry and Molecular Biophysics, Washington University School of Medicine, St. Louis, MO, 63110, USA

*Corresponding author:

Michael J. Greenberg

Department of Biochemistry and Molecular Biophysics

Washington University School of Medicine 660 S. Euclid Ave., Campus Box 8231

St. Louis, MO 63110

Phone: (314) 362-8670

Email: greenberg@wustl.edu

Keywords: single molecule, myosin, ensemble average, change point algorithm, MATLAB 


\section{Abstract}

22 Molecular motors couple chemical transitions to conformational changes that perform

23 mechanical work in a wide variety of biological processes. Disruption of this coupling can

24 lead to diseases, and therefore there is a need to accurately measure mechanochemical coupling in motors in both health and disease. Optical tweezers, with nanometer spatial and millisecond temporal resolution, have provided valuable insights into these processes. However, fluctuations due to Brownian motion can make it difficult to precisely resolve these conformational changes. One powerful analysis technique that has improved our ability to accurately measure mechanochemical coupling in motor proteins is ensemble averaging of individual trajectories. Here, we present a user-friendly

31 computational tool, Software for Precise Analysis of Single Molecules (SPASM), for 32 generating ensemble averages of single-molecule data. This tool utilizes several 33 conceptual advances, including optimized procedures for identifying single-molecule 34 interactions and the implementation of a change point algorithm, to more precisely resolve molecular transitions. Using both simulated and experimental data, we demonstrate that

36 these advances allow for accurate determination of the mechanics and kinetics of the

37 myosin working stroke with a smaller set of data. Importantly, we provide our open source 38 MATLAB-based program with a graphical user interface that enables others to readily apply these advances to the analysis of their own data. 


\section{Statement of Significance}

42 Single molecule optical trapping experiments have given unprecedented insights into the

43 mechanisms of molecular machines. Analysis of these experiments is often challenging

44 because Brownian motion-induced fluctuations introduce noise that can obscure

45 molecular motions. A powerful technique for analyzing these noisy traces is ensemble

46 averaging of individual binding interactions, which can uncover information about the

47 mechanics and kinetics of molecular motions that are typically obscured by Brownian

48 motion. Here, we provide an open source, easy-to-use computational tool, SPASM, with

49 a graphical user interface for ensemble averaging of single molecule data. This

50 computational tool utilizes several conceptual advances that significantly improve the

51 accuracy and resolution of ensemble averages, enabling the generation of high-resolution

52 averages from a smaller number of binding interactions. 


\section{Introduction}

Molecular motors generate force and movement in a wide array of cellular processes, including muscle contraction, packaging of DNA into viral capsids, intracellular transport, DNA damage repair, and cell motility. These motors have complex mechanochemical cycles where chemical transitions are coupled to conformational changes in the protein structure that generate mechanical work. The kinetics and mechanics of these transitions are tuned to the specific molecular role of the motor in the cell, and subtle changes in these properties can lead to an array of diseases (1). Therefore, there is a need for experimental and computational techniques for probing these relationships.

Single-molecule optical trapping techniques, with nanometer spatial and millisecond temporal resolution, have proven to be powerful tools for studying the mechanochemical coupling in motors. One widely used optical trapping technique is the three-bead assay (Fig. 1A) $(2,3)$. In this assay, two beads are held in place by dual-beam optical tweezers. The motor's track (e.g., actin) is strung between these beads and then lowered onto a third, surface-bound bead. This third bead is sparsely coated with motor molecules (e.g., myosin), such that only a single motor interacts with the track at any given time. The positions of the two optically trapped beads are monitored to study the interactions between the motor and the track (Fig. 1B), where motor binding to the track causes both displacement of the beads as well as a reduction in the bead variance. This assay has been applied to study several motor and non-motor systems, including dynein (4), the lac repressor (5), kinesins (6), and several myosin isoforms (7-15). 
Analysis of the individual time-dependent trajectories of motor-induced displacements in the bead positions can provide information about both the mechanics and the kinetics of the motor's mechanochemical cycle. However, it can be difficult to resolve details of these trajectories, as the amplitude of Brownian motion-induced fluctuations in the bead position are frequently larger than the size of motor-induced displacements. One powerful method for extracting high spatial and temporal resolution information from noisy traces is post-synchronization ensemble averaging $(13,16)$. In this method, trajectories from multiple individual binding interactions are aligned and then averaged together, thereby increasing the signal-to-noise ratio. This technique has been applied to successfully identify substeps of the myosin working stroke $(12,13,17)$ and transitions in the ribosome (16) that likely would have been obscured using other analysis methods. While this is a powerful tool for analyzing single-molecule data, there is no software in the public domain that is tailored to performing these calculations, and this has limited the adoption of these tools by many groups.

We have developed a MATLAB-based computational tool, Software for Precise Analysis of Single Molecules (SPASM), with a graphical user interface for the identification and ensemble averaging of single-molecule trajectories. This computational tool utilizes several conceptual advances, including an optimized method for identifying binding interactions from noisy data and improved precision in determining the exact initiation and termination times of binding interactions using a change point algorithm. Using both simulated and experimental data sets, we demonstrate that these advances permit the generation of accurate, high-resolution ensemble averages using fewer individual binding trajectories than were previously required. Our easy-to-use 
99 computational tool includes an intuitive graphical user interface and is offered both as 100 open source code and as a standalone program which does not require full installation of

101 MATLAB. Finally, we provide a user guide, a separate tool for simulating data, and sample 102 data sets to help other researchers apply this tool to their own single-molecule data. 


\section{Methods}

Implementation of the computational tool

The SPASM computational tool, which includes a graphical user interface, was written in MATLAB (MathWorks). The program uses the Signal Processing Toolbox and the Optimization Toolbox, but neither toolbox is required for analysis. The code was tested on MATLAB versions R2017b through R2020a for both Windows and macOS operating systems. Standalone versions of the program for both Windows and macOS were generated using the MATLAB Compiler. For more details, see the Supporting Materials.

\section{Detection of binding interactions}

Binding interactions between a motor and its track in the optical trap can be identified using either a variance $(18)$ or a covariance $(2,19)$ threshold, since the binding of a motor to its track causes a reduction in both the variance and covariance of the two beads (Fig. 2). The covariance between the beads at any time, t, is calculated by:

$$
\operatorname{Cov}_{\mathrm{t}}(\mathrm{A}, \mathrm{B})=\mathrm{E}_{\mathrm{w}_{\mathrm{c}}, \mathrm{t}}[\mathrm{A} * \mathrm{~B}]-\mathrm{E}_{\mathrm{w}_{\mathrm{c}}, \mathrm{t}}[\mathrm{A}] * \mathrm{E}_{\mathrm{w}_{\mathrm{c}}, \mathrm{t}}[\mathrm{B}]
$$

where $A$ is the position of one bead (bead A), B is the position of the other bead (bead $B)$, and $E_{w_{c}, t}[X]$ denotes the mean of $X$ over a window of size $w_{c}$ centered at $t$. Before generating a histogram of covariance values, the covariance is smoothed using a secondorder Savitzky-Golay filter with window size ws to remove high-frequency noise. The values of $w_{c}$ and $w_{s}$ can be optimized using the computational tool. See the Supporting Materials for details. 
A histogram of the filtered covariance between the two beads shows two distinct can be used to determine covariance thresholds for detecting binding interactions (10). methods are discussed in detail in the Results and Discussion. shorter than a user-defined minimum duration are ignored to lower the chance of mistakenly identifying random correlated noise as a binding interaction. Also, any two binding interactions which are separated in time by less than a user-defined minimum separation are ignored to lower the chance of mistakenly identifying random noise as premature detachment between the motor and the track. Note that this filtering takes place after the change points have been located.

Binding interaction alignment using a change point algorithm and the generation of ensemble averages interactions at transitions between the bound and unbound states. Here, we implement a change point algorithm to identify transitions. This algorithm uses maximum likelihood estimation to locate the times, or change points, where changes in both the mean and 
variance of each bead's position have most likely occurred. For each binding interaction identified using covariance thresholds, the algorithm searches for the change points within a window of data. For the $\mathrm{k}^{\text {th }}$ binding interaction, this window spans from

$$
\mathrm{t}_{1}=\mathrm{t}_{\mathrm{k}, \mathrm{start}}-0.49 * \min \left(\mathrm{t}_{\mathrm{k}, \text { end }}-\mathrm{t}_{\mathrm{k}, \mathrm{start}}, \mathrm{t}_{\mathrm{k}, \text { start }}-\mathrm{t}_{\mathrm{k}-1, \mathrm{end}}\right)
$$

to

$$
t_{N}=t_{k, e n d}+0.49 * \min \left(t_{k, \text { end }}-t_{k, \text { start }}, t_{k+1, \text { start }}-t_{k, \text { end }}\right)
$$

where $t_{k, s t a r t}$ and $t_{k, \text { end }}$ denote the beginning and end times of the $\mathrm{k}^{\text {th }}$ interaction as estimated by the covariance threshold method. The window must be wide enough that it includes the entirety of the $\mathrm{k}^{\text {th }}$ interaction but not so wide that it contains part of another interaction. The computational tool automatically searches the default window for change points, but it also allows for manual adjustment of both the search window and the identified change points.

The algorithm considers the average position between beads $A$ and $B$ during this window. For each pair of time points within the window, $\left(t_{i}, t_{j}\right)$, the algorithm calculates the likelihood that these points coincide with changes in the mean and variance of the data. Each pair divides the window into three intervals: $\left[t_{1}, t_{i}\right],\left[t_{i+1}, t_{j}\right]$, and $\left[t_{j+1}, t_{N}\right]$, where $1<\mathrm{i}<\mathrm{j}<\mathrm{N}$. The log-likelihood score, $\mathrm{L}_{\left(\mathrm{t}_{\mathrm{i}}, \mathrm{t}_{\mathrm{j}}\right)}$, assigned to $\left(\mathrm{t}_{\mathrm{i}}, \mathrm{t}_{\mathrm{j}}\right)$ measures how well normal distributions can be fit to these intervals of data:

$$
\left.\mathrm{L}_{\left(\mathrm{t}_{\mathrm{i}}, \mathrm{t}_{\mathrm{j}}\right)}=-\left[\frac{\mathrm{j}-\mathrm{i}}{2} \ln \left(\sigma^{2} \mathrm{t}_{\mathrm{i}+1}, \mathrm{t}_{\mathrm{j}}\right]\right)\right]-\left[\frac{\mathrm{N}-\mathrm{j}+\mathrm{i}}{2} \ln \left(\sigma_{\left[\mathrm{t}_{1}, \mathrm{t}_{\mathrm{i}}\right] \cup\left[\mathrm{t}_{\mathrm{j}+1}, \mathrm{t}_{\mathrm{N}}\right]}\right)\right]
$$

where $\sigma^{2}$ is the variance of the data during the corresponding interval (see the Supporting Materials for the derivation). $\mathrm{L}$ is maximized where the values of $t_{i}$ and $t_{j}$ best divide the 
169 window into three sequences of normally distributed data, and these values of $t_{i}$ and $t_{j}$

170 are then assigned as the change points.

171

After synchronization at the change points, both time forward and time reversed

172 ensemble averages of individual binding interactions are generated from the average of

173 the two beads' positions using well-established methods (16). Shorter-lived binding

174 interactions are extended in time to match the duration of the longest-lived binding

175 interaction. The value of this extension equals the average position of the beads during

176 either the first or last $5 \mathrm{~ms}$ of the binding interaction for the time reversed and time forward

177 averages, respectively.

178

Generation of simulated single-molecule data

To test the accuracy of the program and to aid in the selection of proper window sizes for the analysis of experimental data, we created an additional program to simulate data that resembles single-molecule interactions with user-defined substep sizes and kinetics. The code for this program is provided alongside SPASM so that users can adapt the simulation parameters for their system of interest. Rather than explicitly solving the equations of motion for the optically trapped beads, the parameters used for simulation can be empirically varied until the simulated data matches the experimental data.

187 Trapping data is simulated using a continuous-time Markov jump process in which the motor switches among a baseline detached state and two successive attached states, each with a unique displacement, representing a motor with a two-substep working 190 stroke. The user can set the number of states, the rates of transitioning between the 191 states, and the displacements of each state. High-frequency Gaussian noise is added to 
192 simulate Brownian motion. To simulate mechanical coupling between the beads (i.e., higher covariance), a fraction of the noise in each bead's position, $\mathrm{f}$, is shared between

194 the two beads. When the motor is dissociated from its track, $\mathrm{f}$ is set to a larger number so that the motion of the two beads is correlated. When the motor is bound to the track, $\mathrm{f}$ is set to a lower number, resulting in a lower covariance. Drift in the system is simulated by the addition of low-frequency noise. For additional details, see the Supporting Materials and the provided code.

To test our analysis approach, we generated simulations with well-defined data sets (sets 1-10), each containing 100 binding interactions, to simulate beta cardiac myosin based on previous optical trapping and kinetic measurements $(7,20,21)$. The rate of transitioning from the detached state to the first attached state was set to 0.5 s-1. The rate of transitioning from the first attached state to the second attached state was set to $70 \mathrm{~s}-1$, matching the rate of ADP release (22). The rate of transitioning from the second

208 attached state to the detached state was $4 \mathrm{~s}-1$, matching the rate of ATP-induced actomyosin dissociation at $1 \mu \mathrm{M}$ ATP. The myosin was modeled to have a two-substep working stroke with a $4.7 \mathrm{~nm}$ substep followed by a second substep of $1.9 \mathrm{~nm}(7)$. 
215 was much lower at $5 \mathrm{~s}-1$, and the rate of transitioning from the second attached state to

216

217

220

221

222

223

224

225

226

227

228

229

230

231

232

233

234

235

236

237

the detached state was 2 s-1. As before, the myosin was modeled to have a two-substep working stroke with a $4.7 \mathrm{~nm}$ substep followed by a second substep of $1.9 \mathrm{~nm}$.

With the simulated data, the exact locations of transition points between the bound and unbound states are known, allowing us to test the performance of different analysis methods with regards to: (1) the frequency of false positive binding interactions (i.e., when the bound state is incorrectly detected while the motor is actually unbound), (2) the number of false negative binding interactions (i.e., when the unbound state is incorrectly detected while the motor is actually bound), and (3) the error in determining the correct initiation and termination times of each binding interaction.

To determine the number of false positives, each detected binding interaction was mapped to the nearest overlapping real binding interaction. If a detected binding interaction did not overlap with any real binding interactions, it was counted as a false positive. If multiple detected binding interactions were mapped to the same real binding interaction, all but the closest were also counted as false positives. As we fixed the number of simulated binding interactions within each data set, rather than the total duration of each data set, the data sets typically varied in duration. A longer set of data is expected to result in more false positives, and so the frequency of false positives was calculated by dividing the number of false positives by the duration of the data set. To determine the number of false negatives, each real binding interaction was mapped to the nearest overlapping detected binding interaction. If a real binding interaction did not overlap with any detected binding interactions, it was counted as a false negative. If multiple real binding interactions were mapped to the same detected binding interaction, 
238 all but the closest were also counted as false negatives. The error was calculated as the difference between the computationally identified transition points and the nearest simulated transition points for which the corresponding binding interactions overlapped. frequency of false positives or the mean number of false negatives between the two methods, $p$-values were obtained from the independent two-sample t-test. To test if the median error of the detected transition points was significantly changed with the addition of the change point algorithm, p-values were obtained from the Wilcoxon rank sum test.

251 the known locations of actual simulated binding interactions. To extract parameters from

252 the ensemble averages, exponential curves were fit to each average, yielding estimates

253 for the substep sizes and rates of the simulated data. For each extracted parameter, a 254 Kruskal-Wallis test was used followed by pairwise Wilcoxon rank sum tests to determine 255 p-values.

257 Design of optical trapping apparatus 
261 was rotated by 45 degrees and then separated into vertically and horizontally polarized

262 components to form 2 independent traps. Optical traps were independently steerable

263

264

265

266

267

268

269

270

271

272

273

274

275

276

277

278

279

280

281

282

283

using acoustic optical deflectors (Gooch and Housego) and frequency synthesizer boards under FPGA control (Analog Devices, AD9910 Direct Digital Synthesis evaluation boards). The displacement of the beads from the center of the optical trap was measured at the back focal plane using two quadrant photodiodes (First Sensor). Data were low pass filtered (Frequency Devices) to the Nyquist frequency and digitized on a National Instruments FPGA board (PCle 7852) with simultaneously sampling analog to digital converters. System control was accomplished by custom software written in LabView. 3D stage control was achieved using a piezoelectric stage (Mad City Labs). Fluorescence was illuminated using the output of a $50 \mathrm{~mW} 532$ laser (Crystalaser). Imaging was performed using an EMCCD camera (Andor).

\section{Optical trapping experiments}

Porcine cardiac myosin and actin were purified from cryoground tissue (Pelfreez) as previously described $(25,26)$. Bead coated flow cells were assembled as previously described $(2,7,8)$. All experiments were performed in KMg25 buffer (60 mM MOPS pH 7.0, $25 \mathrm{mM} \mathrm{KCl,} 2 \mathrm{mM}$ EGTA, $4 \mathrm{mM} \mathrm{MgCl}$, $1 \mathrm{mM}$ DTT). All buffers and dilutions were prepared fresh each day. Biotin-labeled actin $(2 \mu \mathrm{M})$ was prepared using $10 \%$ biotin actin (Cytoskeleton) in KMg25 buffer. The mixture was allowed to polymerize for 20 minutes, and then the actin was stabilized using tetramethylrhodamine isothiocyanate-labeled phalloidin. Streptavidin beads (Bangs Labs) were washed in $1 \mathrm{mg} / \mathrm{mL}$ BSA in KMg25 buffer three times. Flow cells were loaded with myosin (4-20 nM in KMg25 with 200 mM 
$284 \mathrm{KCl}$ ) for 5 minutes and then blocked with $1 \mathrm{mg} / \mathrm{mL}$ BSA for 5 minutes. Activation buffer contained KMg25 with the addition of $1 \mu \mathrm{M} \mathrm{ATP,} 192 \mathrm{U} / \mathrm{mL}$ glucose oxidase, $48 \mu \mathrm{g} / \mathrm{mL}$

286 catalase, $1 \mathrm{mg} / \mathrm{mL}$ glucose, and $\sim 25 \mathrm{pM}$ Biotin rhodamine-phalloidin actin. A small

287 amount $(4 \mu \mathrm{L})$ of streptavidin beads were loaded into the flow cell, and the flow cell was

288 sealed with vacuum grease. Trapping experiments were conducted as previously

289 described (2). Two streptavidin beads were optically trapped, forming a bead-actin-bead

290 dumbbell. Trap stiffness was determined by fitting of the power spectral density collected

291 at $20 \mathrm{kHz}$. The bead-actin-bead dumbbell was pretensed to approximately 2-3 pN and 292 then lowered onto a surface bead to search for binding interactions. Approximately 1 in 5

293 beads showed binding interactions. Data were acquired at $2 \mathrm{kHz}$ and filtered to $1 \mathrm{kHz}$. 


\section{Results and Discussion}

295

296

297

298

299

300

301

302

303

304

305

306

307

308

309

310

311

312

313

314

315

316

\section{Ensemble averaging of single-molecule binding interactions}

Ensemble averaging is a powerful method for analyzing single-molecule data, since it can uncover subtle molecular transitions obscured by Brownian motion $(13,16)$. In ensemble averaging, the time-dependent trajectories of individual binding interactions are synchronized and then averaged. While ensemble averaging techniques are broadly applicable, we will focus in this paper on their application to studying the interaction between myosin molecular motors and actin.

Using ensemble averaging of optical trapping data, it has been shown that many myosin isoforms have a two-substep working stroke, where the first substep corresponds to the release of inorganic phosphate and the second substep corresponds to a transition associated with ADP release (Fig. 1C-D) $(7-10,12-14,17,27)$. It is difficult to distinguish the second transition from raw data traces due to Brownian motion. However, ensemble averaging allows for easier visualization of this transition by increasing the signal-to-noise ratio.

One can collect information about both the kinetics and mechanics of the working stroke substeps from the post-synchronized ensemble averaged trajectories of individual binding interactions $(13,16)$. These interactions can be synchronized upon actomyosin attachment and then averaged forward in time or, alternatively, synchronized upon actomyosin detachment and then averaged backward in time (Fig. 1E-F). The magnitude of the initial displacement seen in the time forward averages gives the size of the first substep of the myosin working stroke, a transition which occurs within the dead time of typical optical tweezer instruments. The amplitude of the subsequent exponential rise in 
317 displacement in the time forward averages gives the size of the second substep of the working stroke. The rate of this exponential rise is the rate of transitioning from the first substep to the second substep, and it is associated with ADP release in myosins (13).

320 For the time reversed ensemble averages, the exponential rise in displacement prior to 321 detachment has an amplitude equal to the size of the second substep, and the rate of this 322 exponential gives the rate of transitioning from the second substep to the detached state, a transition which corresponds to ATP-induced actomyosin dissociation (13).

MATLAB-based computational tool for generating ensemble averages

Here, we have generated an easy-to-use MATLAB-based computational tool, SPASM, which finds binding interactions within noisy data, accurately identifies transitions between the bound and unbound states, and then generates ensemble averages. This tool includes several improvements and optimized procedures for both the identification and alignment of binding interactions, which are discussed below. The tool features a graphical user interface for ease of use and is packaged with an accompanying user guide. We provide the code for this tool as well as a compiled executable file that

334 single-molecule data, as well as the sample simulated data sets used in our analysis (see 335 Supporting Materials).

The first step in generating ensemble averages is the identification of binding 
340 the bead-actin-bead dumbbell undergo fluctuations in their position due to Brownian

341 motion (Fig. 2A). The motion of these beads is mechanically coupled through the actin

342 filament, as evidenced by the covariance between their positions (Fig. 2B). When the

343 surface-bound motor binds to the actin filament, it causes several pronounced changes:

344 (1) it reduces the positional variance of each bead's position, (2) it reduces the coupled

345 motion (covariance) of the two trapped beads, and (3) it displaces the mean position of

346 each bead. The majority of analysis methods for identifying binding interactions utilize the

347 changes in the mean position, variance, and/or covariance of the optically trapped beads

348 upon binding to actin $(11,18,19,24,28)$.

One popular method for selecting binding interactions is to set a threshold based

350

351

352

353

354

355

356

357

358 on the variance or covariance of the beads. The choice of using a variance or covariance threshold for binding interaction identification will partially be dictated by the optical trap layout. For systems which only monitor the position of a single bead, one must use a variance threshold for the position of the single bead. For systems where both bead positions are monitored, a covariance threshold is preferred since it is less sensitive to noisy fluctuations in the data. While we focus on the use of our computational tool with a covariance threshold, the same approaches and conclusions will hold true for a variance threshold based on the position of one bead. A version of SPASM that uses a variance threshold is provided (see Supporting Materials).

Our computational tool identifies binding interactions from the change in the covariance between the positions of the two trapped beads that occurs upon myosin binding to actin. SPASM first calculates the covariance over a sliding window in time and then smooths the covariance over a separate window. With properly chosen window 
363 lengths, the histogram of the covariance values reveals two populations (Fig. 2C), where

364 the higher covariance population corresponds to unbound states and the lower population

365 corresponds to bound states (2). One can then select binding interactions based upon

366 thresholds that distinguish between these two populations (see Selection of binding

367 interactions below).

368 The success of this approach depends on the degree of separation between the 369 two peaks in the covariance histogram. If the peaks are not well separated, the analysis

370 is more susceptible to false and/or missed binding interactions. The ability to generate a 371 histogram with two well separated peaks depends partly on the selection of proper

372 window lengths for the calculation and smoothing of the covariance. Optimal values for

373 these parameters, in turn, depend on the kinetics of the myosin's interaction with actin,

374 the compliance of the myosin and/or myosin-surface attachment, the pretension between

375 the optically trapped beads, and the noise in the system. Therefore, the window lengths

376 often need to be determined empirically. If the kinetics of the myosin's transitions are

377 known from other experimental measurements, one can simulate data and select window

378 lengths which optimize analysis of the simulated data (see Supporting Materials). If kinetic

379 information about the myosin's transitions is unknown, it may not be possible to generate

380 meaningful simulated data. In these cases, the window lengths can be determined

381 empirically through the computational tool's graphical user interface, which allows the

382 user to vary the window lengths until a suitable bimodal covariance histogram is achieved. 
Once a suitable covariance histogram with two well-defined peaks has been generated, the next step is to determine proper thresholds for the covariance which will be used to detect binding interactions. One possibility for distinguishing the bound state from the unbound state is to use a single covariance threshold located at the minimum value between the two peaks of the covariance histogram (10). Here, detected interactions start when the covariance drops below this threshold value, and they end when the covariance rises back above this threshold value (Fig. 2D). Alternatively, one could identify the binding interactions using a set of two different covariance thresholds, located at the two peaks of the covariance histogram. In this 'peak-to-peak' approach, a binding interaction is considered to start when the covariance drops from the threshold defined by the unbound peak to the threshold defined by the bound peak. Likewise, a binding interaction is considered to end when the covariance rises from the threshold defined by the bound peak to the threshold defined by the unbound peak (Fig. 2D). accurately detect simulated binding interactions between actin and cardiac myosin. Interactions were simulated using a continuous-time Markov jump process with kinetics and mechanics based on previously measured parameters for ventricular cardiac myosin $402(7,21,22)$ (see Materials and Methods for details). With simulated data, the exact locations of the binding interactions are known, allowing for easy comparison between 404 the simulated interactions and the interactions detected by the computational tool using 405 either method (Fig. 2D). 
408 the covariance histogram, locate the peaks and minimum of the histogram, and identify binding interactions using either the single threshold method or the peak-to-peak method. When we used a single threshold to identify binding interactions, we correctly detected $41180+/-4$ of the 100 binding interactions on average, and we incorrectly detected $4+/-1$

412 false positive binding interactions per 100 seconds of data, on average (Table 1). The 413 reported errors are standard deviations. When we used the peak-to-peak method to 414 identify binding interactions, we correctly detected $65+/-5$ of the 100 binding interactions 415 on average, and we did not detect any false positive binding interactions. Although the peak-to-peak method misses a greater number of binding interactions, the false positive

417 rate is lower for this method $(p<0.001)$.

A single threshold could work well for selecting binding interactions if the two populations of the histogram are sufficiently distinct. However, it is often not possible to obtain sufficient separation between the peaks due to factors that lower the signal-tonoise ratio (e.g., system noise, insufficient pretension between the beads, fast binding

422 kinetics). In these cases, this single threshold approach is prone to identifying false positive interactions, where the covariance crosses the threshold even though the

424 actomyosin has remained in an unbound state. These false positive binding interactions

425 do not generate a net displacement in the optical trap, and so their inclusion in the ensemble averages is expected to lead to an underestimation of the true size of the

427 working stroke. A methodology has been developed which attempts to correct for these 428 false positive interactions through the use of normalization factors (10). Alternatively, as 429 the vast majority of these false positive interactions arise due to either Brownian motion 430 (or system noise) induced rapid downward spikes in the covariance (which lead to very 
431 short detected interactions) or rapid upward spikes in the covariance (which lead to

432 multiple detected interactions in quick succession), it is possible to avoid these false

433 positive interactions through the use of temporal filters that exclude interactions which are

434 too short or pairs of interactions which are too close to one another. However, it is not

435 always easy to determine appropriate values for these temporal filters. Further, the use

436 of these temporal filters may lead to the exclusion of many correctly identified binding

437 interactions. When we used optimized values for these filters to exclude all of the false

438 positive interactions that were detected by the single threshold method, we were left with

439 fewer interactions than were detected by the peak-to-peak method (Fig. S2).

much stricter than with the single threshold method, and the number of identified false

442 positive binding interactions is expected to decrease while the number of missed, short-

443 lived binding interactions increases. Unlike the inclusion of false positive interactions, the

exclusion of these missed binding interactions does not adversely affect the size or shape

445 of the ensemble averages. Although we demonstrate that the peak-to-peak method 446 performs better in data traces with moderate separation between the peaks of the

447 covariance histogram, some experimental data might have better peak separation. In this

448 case, the single threshold method would be preferable since it maximizes the number of

449 captured binding interactions. The computational tool allows the user to try both methods, 450 and it automatically determines appropriate values for the thresholds. 
After binding interactions are identified, they must be precisely aligned at the

454 transitions between the bound and unbound states to generate accurate ensemble

455 averages. The most critical step in aligning these interactions is the careful determination

456 of when exactly a transition occurs. Inadequate determination of these transitions will lead

457 to inaccurate measurements of the substep sizes and/or kinetics. Several methods have

458 been applied to locate transitions in single-molecule data traces, including Hidden Markov

459 Models (28) and step finding algorithms (29), but a frequently used method for post-

460 synchronization is to align the binding interactions based on the same thresholds used to

461 identify the binding interactions $(2,10,13)$.

To test the abilities of the single threshold and peak-to-peak methods to accurately

463 identify the transitions, we used the same 10 simulated data sets containing 100

464 transitions each, as described previously (sets 1-10). When we used a single threshold

465 to identify transition times, we found that the detected attachment times occurred 28.2

466 (95\% confidence intervals: $+13.8,-21.7)$ milliseconds after the actual attachment times,

467 on average (Table 2), and the detected detachment times occurred $28.6(+11.9,-19.1)$

468 milliseconds before the actual detachment times, on average. On the other hand, when

469 we used the peak-to-peak method to identify transitions, we found that the detected

470 attachment times occurred $55.5(+195.5,-69.0)$ milliseconds before the actual attachment

471 times, on average, and the detected detachment times occurred $50.4(+188.1,-64.9)$

472 milliseconds after the actual detachment times, on average. Taken together, the single

473 threshold method has better temporal resolution when identifying transitions between the

474 bound and unbound states. 
When binding interactions are aligned based on the covariance thresholds, it is assumed that the covariance drops and rises in conjunction with transitions between the

477 bound and unbound states. With the single threshold method, this is a fairly reasonable

478 assumption, explaining why it outperforms the peak-to-peak method. Each true transition

479 point separates more highly correlated bead motion (i.e., the unbound state) from less

480 highly correlated bead motion (i.e., the bound state). The covariance is calculated over a

481 window, so when the covariance window is centered at a transition point, the window will

482 include equal amounts of more highly and less highly correlated data. The covariance at

483 the transition point should then lie at some intermediate value between the two peaks of

484 the covariance histogram. However, the single threshold method is not perfect at locating

485 the transition points. First, while the value of the covariance at a transition point will likely

486 be near the minimum value between the two peaks of the covariance histogram, there is

487 no guarantee that it will lie exactly at this minimum value. Additionally, synchronized large-

488 scale movement of both beads due to the myosin's power stroke can produce transient spikes in the covariance value during transitions, and these spikes can potentially decrease the accuracy of the single threshold method in identifying exact transition times.

The peak-to-peak method produced poorer alignment than the single threshold

method. When the peak-to-peak method is used to identify transitions, it is assumed that transitions occur when the covariance crosses the upper threshold, defined by the

494 position of the unbound peak. This is inherently less accurate for estimating transition 495 points than the single threshold method. A window of data which has a covariance value that is similar to the value of the unbound peak contains primarily correlated data and, 497 therefore, it is unlikely that the center of this window is near the actual transition point. In 
498 fact, the calculated transition point using the peak-to-peak method would be expected to 499 deviate from the actual transition point by at least half the window size.

Taken together, our data show that when binding interactions are synchronized

501

502

503

504

505

506

507

508

509

510

511

512 improve our ability to locate the transition times of each binding interaction, we

513 implemented a change point algorithm (see Materials and Methods for details). Change

514 point algorithms have been used in step finding for transitions in biological processes,

515 where the algorithm identifies the most likely times in which there was a change in a

516 parameter such as motor position or rotation of the myosin lever arm $(29,30)$. We have

517 adapted the change point algorithm for the three-bead assay, where we search for the

518 most likely transition times based on changes in both the mean and the variance of the

519 bead positions, as both of these parameters differ between the bound and unbound states

520

(Fig. 3A). For each binding interaction identified by the covariance threshold method (Fig. 
521 3B), our algorithm examines the positions of the trapped beads in a window surrounding 522 that interaction and finds the two points (i.e., binding initiation and detachment) within this

523 window that most likely represent transitions in the mean and variance of the data (Fig.

524 3C; see Methods for details).

525

To test the ability of the change point algorithm to accurately identify transition

526 times, we again analyzed the same 10 sets of simulated data described above (sets 1-

527 10). We found that the attachment times detected by the change point algorithm occurred

$0.5(+9.0,-5.5)$ milliseconds after the actual attachment times, on average (Table 2), and

529 the detachment times detected by the change point algorithm occurred $0.7(+4.8,-4.2)$

530 milliseconds after the actual detachment times, on average (Table 2). Statistical testing

531 demonstrates that the change point algorithm outperforms both the single threshold

532 method ( start $<0.001$, pend $<0.001$ ) and the peak-to-peak method ( start $<0.001$, Pend $<$

533 0.001) in identifying transition times. As our simulated data were generated with a

534 sampling frequency of $2 \mathrm{kHz}$, these average errors of about $0.5 \mathrm{~ms}$ indicate that the

535 change point algorithm was typically correct within 1 point. It is possible that a higher

536 sampling frequency would further increase the accuracy.

To explore the ability of these three methods to accurately identify transition points,

538 we generated cumulative distributions of the differences between the detected transition

539 times and the actual simulated transition times for both the initiation and termination of

540 the binding interactions (Fig. 4). Here, the width of the distribution reveals the precision

541 of the corresponding method, while the sign and magnitude of the average error reveals

542 the systematic bias of that method. As expected, the cumulative distributions of errors

543 generated from the peak-to-peak method are wide, indicating low precision at identifying 
544 the transitions, while the distributions generated from the single threshold method are

545

546

547

548

549

550

551

552

553

554

555

556

557

558

559

560

561

562

563

564

565 narrower, indicating higher precision. The distributions generated from the change point algorithm are very narrow, and the mean error is close to 0 . This indicates that the change point algorithm is very precise and has lower systematic bias than either the single threshold or peak-to-peak method.

\section{Comparison of ensemble averages generated using different methods}

To test our predictions about the relative accuracy of the ensemble averages when using each method of analysis, we generated ensemble averages from the 10 sets of simulated data studied previously (sets 1-10). First, we generated ensemble averages using the actual locations of all 1000 simulated binding interactions to align the binding interactions (Fig. 5A-B, real). We also generated ensemble averages for each of the 10 sets of data, using the actual locations of the 100 simulated binding interactions within each set. Exponential curves were fit to each of these averages to estimate the substep sizes and rates of the simulated myosin working stroke (Fig. 5C-F, real; Table $\mathbf{3}$ ). The magnitude of substep 1 estimated from the time forward averages was 4.7 (95\% confidence intervals: $+0.4,-0.4) \mathrm{nm}$, on average, while the magnitude of the total step estimated from the time forward averages was $6.4(+0.2,-0.2) \mathrm{nm}$, on average. The magnitude of substep 1 estimated from the time reversed averages was $5.7(+0.2,-0.3)$ $\mathrm{nm}$, on average, while the magnitude of the total step estimated from the time reversed averages was $6.5(+0.1,-0.2) \mathrm{nm}$, on average. The estimated rate of transitioning from the first substep to the second substep $\left(\mathrm{kf}_{\mathrm{f}}\right)$ was $68.7(+15.8,-20.9) \mathrm{s}-1$, and the estimated 
566 rate of transitioning from the second substep to the detached state $\left(\mathrm{kr}_{\mathrm{r}}\right)$ was $4.3(+2.2,-$

567 1.9) S-1.

We then used either the single threshold method or the peak-to-peak method to detect binding interactions within each data set. When the single threshold method was used to detect binding interactions, we applied a filter to ignore any detected interactions

571 which were shorter than $77 \mathrm{~ms}$ or within $63 \mathrm{~ms}$ of another detected interaction, to avoid 572 including false positive interactions (Fig. S2; Fig. S3 shows the effect of including these

573 false positive binding interactions). To identify transitions between the bound and unbound states for each interaction, we either included or omitted the change point

575 algorithm. For each of these analysis methods, we used the binding interactions and

576 transitions detected over all 10 data sets to generate ensemble averages (Fig. 5A-B). As

577 before, we also generated ensemble averages from the binding interactions detected

578 within each of the 10 sets of data, and exponential curves were fit to each average to estimate the substep sizes and rates of the simulated myosin working stroke (Fig. 5C-F;

Table 3). As expected, using the change point algorithm to align the binding interactions resulted in the most accurate estimates.

When the peak-to-peak method was used to both detect and align the binding

583 interactions, the ensemble averages were misshapen (Fig. 5, PTP). The time forward 584 average, for example, includes the characteristic increase in displacement but then drops.

585 This drop is due to the fact that the binding interaction termination times detected by the 586 peak-to-peak method often came after the actual termination times, leading to the 587 inclusion of baseline data at the end of the time forward average. The time forward 588 average also appears to start too late, as the peak-to-peak method typically guesses that 
589 binding initiation times occur before they actually do (Fig. 4). Exponential curves were 590 very poorly fit to these ensemble averages.

When the single threshold method was used to both detect and align the binding

592 interactions, the ensemble averages had better overall shape (Fig. 5, ST). However,

593 similar to the averages generated with the peak-to-peak method, misalignment among

594 the individual trajectories resulted in very gradual transitions between the bound and 595 unbound states. The time forward average, for example, appears to start too early, as the 596 single threshold method typically guesses that binding initiation times occur after they 597 actually do (Fig. 4).

When the change point algorithm was used to align the binding interactions, the ensemble averages featured much sharper transitions (Fig. 5, PTP/CP and ST/CP). 600 However, very sharp spikes in displacement occur at the transition times (Fig. 5A-B, 601 PTP/CP and ST/CP). Brownian motion-driven fluctuations in the bead positions can cause changes in the data from one point to the next which are not due to transitions between the bound and unbound states. If such noise happens to occur near a real 604 transition point, it offers an attractive candidate for the change point, and the change point 605 algorithm may choose that point instead of the less pronounced yet correct transition time.

606 However, we have shown that the transition times estimated by the change point 607 algorithm are within 1 to 2 points of the actual simulated transition times, on average 608 (Table 2; Fig. 4), and the resulting ensemble averages are very accurate. Appropriate fits 609 can be obtained by omitting these spikes from the fitted data. 
612 5B-C; Table 3). To generate the time reversed ensemble average, short-lived binding

613 interactions are extended in time to match the duration of the longest-lived binding

614 interaction, and the value of this extension equals the average position of the beads

615 during the first $5 \mathrm{~ms}$ of the binding interaction. The rate of transitioning from the first

616 substep to the second substep in our simulated data was $70 \mathrm{~s}-1$, matching the rate of ADP

617 release for beta cardiac myosin (22). Because of this fast rate, a large number of

618 transitions to the second substep occur before the $5 \mathrm{~ms}$ used to generate the extensions,

619 leading to inaccurate extension values. The proportion of binding interactions which are

620

expected to transition to the second substep within the first $5 \mathrm{~ms}$ is given by the integral

621 of the probability density function:

622

$$
\text { proportion of substeps missed }=\int_{0}^{0.005} \mathrm{ke}^{-\mathrm{kt}} \mathrm{dt}
$$

For a rate of $70 \mathrm{~s}-1$, this proportion is equal to about $30 \%$, and this will lead to an overestimate of the size of the first substep. A possible fix is to shorten the $5 \mathrm{~ms}$ window used for calculating the extensions, but it then becomes crucial that the binding initiation times are determined with high accuracy. Neither the single threshold method nor the peak-to-peak method have sufficient resolution to accurately determine the exact initiation times (Fig. 4). Even the change point algorithm, which we have shown to have an average error of about $0.5 \mathrm{~ms}$, would be insufficient for generating the time reversed ensemble averages of interactions with very fast kinetics. It is possible that this could be improved with faster data sampling. In the case of transitions with slower kinetics, this problem is easily avoided. When we simulated 1000 binding interactions using much slower rates ( $\mathrm{kf}_{\mathrm{f}}$ of $5 \mathrm{~s}-1$ and $\mathrm{kr}$ of $2 \mathrm{~s}-1$, sets 11-20), we were able to generate time forward and time 
Performance of the computational tool to analyze experimental data

To test the ability of the computational tool on real experimental data, we conducted optical trapping experiments using ventricular myosin at $1 \mu \mathrm{M}$ ATP (Fig. 6).

640 molecules. Binding interactions were identified using the peak-to-peak method, and 641 transition points were identified using the change point algorithm. The SPASM

642 computational tool was used to generate cumulative distributions of individual binding 643 interactions (Fig. 6B). The cumulative distributions of the attachment durations is well fit 644 by a single exponential function. This exponential rate gives the rate of actomyosin 645 detachment, and it has a value of $4.7 \mathrm{~s}-1$, which is consistent with the expected rate of 646 ATP-induced actomyosin dissociation at $1 \mu \mathrm{M}$ ATP (22). The cumulative distribution of

647 total working stroke displacements is well fit by a single normal distribution (indicating 648 likely single molecule conditions), with a mean of displacement of $6.3 \mathrm{~nm}$ and a standard deviation of $9.2 \mathrm{~nm}$. This is consistent with previous measurements of the cardiac myosin 650 working stroke $(7,21)$. Ensemble averages (Fig. 6C) reveal that, consistent with previous measurements $(7,21)$, ventricular cardiac myosin has a two-substep working stroke with

652 a first substep of $4.4 \mathrm{~nm}$ and a total displacement of $6.4 \mathrm{~nm}$. The time forward averages 653 have a rate of $74 \mathrm{~S}-1$, which is consistent with the rate of ADP release, and the time 654 reversed averages have a rate of $3.2 \mathrm{~S}-1$, which is consistent with the rate of ATP-induced 655 actomyosin dissociation at $1 \mu \mathrm{M}$ ATP (22). Taken together, our computational tool can 656 generate accurate ensemble averages with sharp transitions from a relatively small set 657 of experimental data. 


\section{Broader applicability of the approach}

The methods presented in this paper were applied to study actomyosin. As noted

661 previously, the three-bead assay has been used to explore many different single-

662 molecule systems, including dynein, the lac repressor, and kinesins. Moreover, the

663 general ideas behind our computational tool are broadly applicable to any set of data

664 containing well-defined populations which can be distinguished through some aspect of

665 the data. One such possibility is data obtained from single-molecule FRET experiments.

666 In the Supporting Materials, we describe how to adapt the change point algorithm to

667 systems where the desired change points occur in data with different distributions.

668

669

Limitations

There are a number of limitations accompanying our computational tool and the

671 methods we use to analyze our data. While the covariance between the position of each

672 trapped bead in the three-bead assay is very helpful for locating binding interactions

673 under many circumstances, it does have drawbacks. The covariance is calculated over a

674 window, and therefore it does not always drop enough during short-lived binding

675 interactions to register as a genuine binding interaction. Furthermore, depending on the

676 quality of the data, it may be difficult or even impossible to obtain a covariance histogram

677 with two distinct populations. This could stem from system compliances. One benefit of 678 the peak-to-peak method is that the covariance histogram populations do not need to be

679 completely separated to avoid false positive binding interactions, but a certain degree of 680 separation is needed to make the covariance useful. Additionally, analysis is dependent 
681 on many parameters, including the window sizes used to calculate and smooth the 682 covariance, and it can be difficult to choose appropriate values for these parameters for

683 a given experimental system. The computational tool includes features which allow the 684 user to correct for these drawbacks when they are encountered. Finally, as evidenced by 685 the ensemble averages generated from our simulated data (Fig. 5), ensemble averaging 686 has limitations for estimating the rates and substep sizes for transitions with very fast 687 kinetics.

688

689

Summary

Here, we developed a computational tool, SPASM, for the detection and alignment of single-molecule binding interactions and for the generation of ensemble averages

692 which can reveal characteristics about the data that are often obscured by noise. We 693 show that it can be advantageous to use separate techniques for the detection and 694 alignment of binding interactions. Specifically, we show that the addition of a change point 695 algorithm to identify transition times can generate precise ensemble averages with 696 improved alignment. We offer the computational tool, with an intuitive graphical user 697 interface, along with a user guide so that the reader can apply these methods to their own 698 data. 


\section{Acknowledgements:}

700 Funding for this project was provided by the National Institutes of Health (R01HL141086

701 to M.J.G., T32EB018266 to S.R.C.).

702

703

Conflict of interest statement:

704

All experiments were conducted in the absence of any commercial or financial relationships that could be construed as a potential conflict of interest.

706

707

Author contributions:

T.B. wrote the computational tool, simulated data, and analyzed data. W.T.S. built the optical trap and wrote software for data acquisition. S.R.C. collected optical trapping data. M.J.G. wrote code for the simulator and analyzed data. T.B. and M.J.G. wrote the first

711 draft of the paper, and all authors contributed to the final draft.

Code availability:

714 The SPASM computational tool can be found at:

715 https://github.com/GreenbergLab/SPASM

717 This repository includes the open source code for SPASM (SPASM.m), compiled 718 versions for Windows (SPASM_Windows.exe) and macOS (SPASM_macOS.app.zip), 719 a versions of the program which analyze only one trapped bead and uses variance 720 thresholds rather than covariance thresholds (SPASM_one_bead.m, 721 SPASM_one_bead_Windows.exe, SPASM_one_bead_macOS.app.zip), MATLAB 
bioRxiv preprint doi: https://doi.org/10.1101/2020.08.10.241752; this version posted August 10,2020 . The copyright holder for this preprint (which was not certified by peer review) is the author/funder, who has granted bioRxiv a license to display the preprint in perpetuity. It is made available under aCC-BY-NC-ND 4.0 International license.

722 code to generate simulated data (simulator.m), a user guide for the aforementioned

723 components (User_Guide.pdf), and the simulated data sets analyzed in this paper (sets

724 1-20).

725 


\section{References}

727 1. Spudich, J. A. 2014. Hypertrophic and dilated cardiomyopathy: four decades of basic research on

728 muscle lead to potential therapeutic approaches to these devastating genetic diseases.

729

730 Biophysical journal 106:1236-1249.

731

732

2. Greenberg, M. J., H. Shuman, and E. M. Ostap. 2017. Measuring the Kinetic and Mechanical Properties of Non-processive Myosins Using Optical Tweezers. Methods Mol Biol 1486:483-509.

3. Finer, J. T., R. M. Simmons, and J. A. Spudich. 1994. Single myosin molecule mechanics: piconewton forces and nanometre steps. Nature 368:113-119.

733

734 Walter, W. J., M. P. Koonce, B. Brenner, and W. Steffen. 2012. Two independent switches regulate cytoplasmic dynein's processivity and directionality. Proceedings of the National Academy of Sciences of the United States of America 109:5289-5293. myosin working stroke. Nature methods 9:1013-1019.

6. Pyrpassopoulos, S., H. Shuman, and E. M. Ostap. 2020. Modulation of Kinesin's Load-Bearing Capacity by Force Geometry and the Microtubule Track. Biophysical journal 118:243-253.

743

744

7. Greenberg, M. J., H. Shuman, and E. M. Ostap. 2014. Inherent force-dependent properties of betacardiac myosin contribute to the force-velocity relationship of cardiac muscle. Biophysical journal 107:L41-L44.

8. Greenberg, M. J., T. Lin, Y. E. Goldman, H. Shuman, and E. M. Ostap. 2012. Myosin IC generates power over a range of loads via a new tension-sensing mechanism. Proceedings of the National Academy of Sciences of the United States of America 109:E2433-2440.

9. Lewis, J. H., M. J. Greenberg, J. M. Laakso, H. Shuman, and E. M. Ostap. 2012. Calcium regulation of myosin-I tension sensing. Biophysical journal 102:2799-2807.

10. Laakso, J. M., J. H. Lewis, H. Shuman, and E. M. Ostap. 2008. Myosin I can act as a molecular force sensor. Science 321:133-136.

11. Guilford, W. H., D. E. Dupuis, G. Kennedy, J. Wu, J. B. Patlak, and D. M. Warshaw. 1997. Smooth muscle and skeletal muscle myosins produce similar unitary forces and displacements in the laser trap. Biophysical journal 72:1006-1021.

12. Veigel, C., F. Wang, M. L. Bartoo, J. R. Sellers, and J. E. Molloy. 2002. The gated gait of the processive molecular motor, myosin $\mathrm{V}$. Nature cell biology 4:59-65.

13. Veigel, C., L. M. Coluccio, J. D. Jontes, J. C. Sparrow, R. A. Milligan, and J. E. Molloy. 1999. The motor protein myosin-I produces its working stroke in two steps. Nature 398:530-533.

14. Veigel, C., M. L. Bartoo, D. C. White, J. C. Sparrow, and J. E. Molloy. 1998. The stiffness of rabbit skeletal actomyosin cross-bridges determined with an optical tweezers transducer. Biophysical journal 75:1424-1438.

762

15. Chuan, P., J. A. Spudich, and A. R. Dunn. 2011. Robust mechanosensing and tension generation by myosin VI. Journal of molecular biology 405:105-112.

16. Chen, C., M. J. Greenberg, J. M. Laakso, E. M. Ostap, Y. E. Goldman, and H. Shuman. 2012. Kinetic schemes for post-synchronized single molecule dynamics. Biophysical journal 102:L23-25.

765

766

17. Capitanio, M., M. Canepari, P. Cacciafesta, V. Lombardi, R. Cicchi, M. Maffei, F. S. Pavone, and R. Bottinelli. 2006. Two independent mechanical events in the interaction cycle of skeletal muscle myosin with actin. Proceedings of the National Academy of Sciences of the United States of America 103:87-92.

771

18. Molloy, J. E., J. E. Burns, J. Kendrick-Jones, R. T. Tregear, and D. C. White. 1995. Movement and force produced by a single myosin head. Nature 378:209-212. 
804

805

806

807

808

19. Mehta, A. D., J. T. Finer, and J. A. Spudich. 1997. Detection of single-molecule interactions using correlated thermal diffusion. Proceedings of the National Academy of Sciences of the United States of America 94:7927-7931.

20. Sung, J., S. Nag, K. I. Mortensen, C. L. Vestergaard, S. Sutton, K. Ruppel, H. Flyvbjerg, and J. A. Spudich. 2015. Harmonic force spectroscopy measures load-dependent kinetics of individual human beta-cardiac myosin molecules. Nat Commun 6:7931.

21. Woody, M. S., M. J. Greenberg, B. Barua, D. A. Winkelmann, Y. E. Goldman, and E. M. Ostap. 2018. Positive cardiac inotrope omecamtiv mecarbil activates muscle despite suppressing the myosin working stroke. Nat Commun 9:3838.

22. Deacon, J. C., M. J. Bloemink, H. Rezavandi, M. A. Geeves, and L. A. Leinwand. 2012. Identification of functional differences between recombinant human alpha and beta cardiac myosin motors. Cellular and molecular life sciences: CMLS 69:2261-2277.

23. Sung, J., S. Sivaramakrishnan, A. R. Dunn, and J. A. Spudich. 2010. Single-molecule dual-beam optical trap analysis of protein structure and function. Methods in enzymology 475:321-375.

24. Takagi, Y., E. E. Homsher, Y. E. Goldman, and H. Shuman. 2006. Force generation in single conventional actomyosin complexes under high dynamic load. Biophysical journal 90:1295-1307.

25. Barrick, S. K., S. R. Clippinger, L. Greenberg, and M. J. Greenberg. 2019. Computational Tool to Study Perturbations in Muscle Regulation and Its Application to Heart Disease. Biophysical journal 116:2246-2252.

26. Clippinger, S. R., P. E. Cloonan, L. Greenberg, M. Ernst, W. T. Stump, and M. J. Greenberg. 2019. Disrupted mechanobiology links the molecular and cellular phenotypes in familial dilated cardiomyopathy. Proceedings of the National Academy of Sciences of the United States of America 116:17831-17840.

27. Takagi, Y., R. E. Farrow, N. Billington, A. Nagy, C. Batters, Y. Yang, J. R. Sellers, and J. E. Molloy. 2014. Myosin-10 produces its power-stroke in two phases and moves processively along a single actin filament under low load. Proceedings of the National Academy of Sciences of the United States of America 111:E1833-1842.

28. Smith, D. A., W. Steffen, R. M. Simmons, and J. Sleep. 2001. Hidden-Markov methods for the analysis of single-molecule actomyosin displacement data: the variance-Hidden-Markov method. Biophysical journal 81:2795-2816.

29. Kerssemakers, J. W., E. L. Munteanu, L. Laan, T. L. Noetzel, M. E. Janson, and M. Dogterom. 2006. Assembly dynamics of microtubules at molecular resolution. Nature 442:709-712.

30. Beausang, J. F., Y. E. Goldman, and P. C. Nelson. 2011. Changepoint analysis for single-molecule polarized total internal reflection fluorescence microscopy experiments. Methods in enzymology 487:431-463. 


\section{Figure legends:}

Figure 1. Ensemble averaging of optical trapping data enables the study of mechanochemical coupling. (A) Diagram of the three-bead assay, where an actin filament strung between the two optically trapped beads is lowered onto a third surfacebound bead that is sparsely coated with myosin. (B) Single-molecule binding interactions between cardiac myosin and actin at $1 \mu \mathrm{M}$ ATP recorded in the optical trap. The average position between the optically trapped beads is plotted as a function of time, with blue horizontal bars indicating detected binding interactions. The mean position and variance of the beads change upon binding. Brownian motion obscures the second substep of the working stroke. (C) Schematic showing the two substeps of the myosin working stroke. (D) Idealized trace showing the position over time of a motor with a two-substep working stroke without Brownian motion. (E) Procedure for generating time forward ensemble averages from individual binding interactions. Individual trajectories are aligned at the initiation of binding and averaged forward in time (black line), and the average is fit with a single exponential function (red). The y-offset and amplitude of this exponential provide estimates of the average size of the first and second substeps, respectively. The rate of this exponential gives the rate of transitioning from the first substep to the second substep. (F) Procedure for generating time reversed ensemble averages from individual binding interactions. Individual trajectories are aligned upon dissociation and averaged backwards in time (black), and the average is fit with a single exponential function (red). The y-offset and amplitude of this exponential provide estimates of the average size of the total step and the second substep, respectively. The rate of this exponential gives the rate of transitioning from the second substep to the detached state. 

histogram of covariance values shows two distinct populations which correspond to the two populations (green). In the peak-to-peak method, two thresholds are placed, one at the peak of each population (red), and a binding interaction is detected when the covariance transitions from one threshold to the other threshold. (D) Simulated binding interactions detected by the peak-to-peak method (red), binding interactions detected by the single threshold method (green), and actual simulated binding interactions (blue). The single threshold is more susceptible to false positive interactions (circled). The peak-topeak method is more susceptible to false negative interactions (boxed).

Figure 3. The change point algorithm more precisely identifies transitions between

bound and unbound states. (A) Simulated optical trapping data showing the average position between the optically trapped beads over time during a binding interaction. Data obtained during the bound state (purple) are drawn from a normal distribution with a shifted mean and a lower variance when compared to data obtained during the unbound state (black). The change point algorithm seeks to find the time points which best separate 855 the two distributions. The locations of the actual simulated transitions are marked with 
856 blue vertical lines. (B) Calculated covariance of the bead positions during the simulated 857 binding interaction in $(\mathrm{A})$. The attachment and detachment times identified by the single 858 threshold (green) and the peak-to-peak (red) methods are shown with dashed vertical

859 lines. The actual transitions are marked with solid blue vertical lines. (C) The change point 860 algorithm determines the likelihood that any two points within an extended search window are the two transition points. (left) Plot of the likelihood assigned to each pair of points within the search window, viewed from the side (see Materials and Methods for details). The change points, which occur when the likelihood is maximized, are shown with dashed yellow vertical lines, while the actual transitions are marked with solid blue vertical lines. (right) The likelihood viewed from above. Regions of yellow correspond to higher

866 likelihood, while regions of dark blue correspond to lower likelihood. The two change 867 points are marked with solid black lines.

Figure 4. The change point algorithm minimizes the error when detecting the locations of transitions. The error was calculated as the difference between the detected binding times and the actual simulated binding times for simulated data (sets 1-

872 10). (left) Cumulative distributions of the errors in determining the binding initiation times 873 using the peak-to-peak method (red), the single threshold method (green), and the 874 change point algorithm (yellow). Statistical comparisons can be found in Table 2. (right) 875 Cumulative distributions of the errors when determining the binding termination times 876 using the peak-to-peak method (red), the single threshold method (green), and the 877 change point algorithm (yellow). 
879 Figure 5. Ensemble averages of simulated binding interactions. 10 sets of data were 880 simulated, each containing 100 binding interactions (sets 1-10). Interactions were 881 detected using either the peak-to-peak (PTP) or the single threshold (ST) method, and 882 interactions were aligned using either the transitions estimated by the covariance 883 threshold method or the change points identified by the change point algorithm (CP). (A)

884 (left) For each analysis method, all detected binding interactions were aligned at the 885 estimated initiation times and averaged together to generate time forward ensemble averages. (right) For each analysis method, all detected binding interactions were aligned at the estimated termination times and averaged together to generate time reversed ensemble averages. Also shown are the time forward and time reversed ensemble averages generated from the known locations of the actual simulated binding interactions (blue, real). (B) A zoomed in view of the boxed segments of the ensemble averages in $A$ highlights the misalignment in the averages when the change point algorithm is omitted.

892 (C-F) For each of the 10 simulated sets of data containing 100 binding interactions, ensemble averages were generated and fit with single exponential functions. The substep sizes and rates of the simulated myosin working stroke were estimated from the exponential fits. Box plots show the estimated parameters for each analysis method.

896 Outliers are indicated by red dots. The substep sizes were estimated from both the time 897 forward $(f)$ and the time reversed $(r)$ ensemble averages. Horizontal dashed lines show 898 the values of the simulated parameters. Statistical analysis for each parameter can be 899 found in Table 3. 
901 Figure 6. Ensemble averages of experimental optical trapping data. The kinetics and

902 mechanics of cardiac myosin in $1 \mu \mathrm{M}$ ATP were measured using the three-bead assay.

903 (A) Experimental data trace showing the displacement (D) and covariance (C). (B)

904 Cumulative distributions for the (left) binding interaction durations and (right) total working

905 stroke displacements. The peak-to-peak method was used to detect binding interactions.

906 Red lines show the cumulative fits based on (left) exponential and (right) normal

907 distributions. The characteristic rate obtained from the fit to the distribution of attachment

908 durations gives a detachment rate equal to $4.7 \mathrm{~s}-1$, which is consistent with the expected 909 rate of ATP-induced actomyosin dissociation at $1 \mu \mathrm{M}$ ATP. The distribution of total step

910 sizes has a mean of $6.3 \mathrm{~nm}$ and a standard deviation of $9.2 \mathrm{~nm}$. (C) The change point 911 algorithm was used to align the interactions identified using the peak-to-peak method. A 912 total of 66 binding interactions from 5 molecules were analyzed. The resulting ensemble 913 averages have estimated substep sizes of $4.4 \mathrm{~nm}$ and $2.0 \mathrm{~nm}$. The estimated time forward 914 rate is $74 \mathrm{~s}-1$, and the estimated time reversed rate is $3.2 \mathrm{~s}-1$. These values are consistent 915 with previous measurements using a much larger data set, and they agree well with the 916 previously measured rates of ADP release and ATP-induced dissociation $1 \mu \mathrm{M}$ ATP. 
Table legends:

Table 1. Detection of binding interactions using either the single or peak-to-peak

covariance threshold method. Average number of correctly identified binding

922 interactions and frequency of false positive binding interactions detected with the single

923 threshold method and peak-to-peak method for 10 data sets, each containing 100 924 simulated binding interactions (sets 1-10). Calculated values were rounded to the nearest 925 whole number.

Table 2. The change point algorithm minimizes the error when detecting the

928 locations of transitions. Mean and 95\% confidence intervals for the error when detecting transitions within simulated data sets 1-10 with the single threshold method, the peak-to-peak method, and the change point algorithm. When estimating the binding initiation times, 645 of 1000 transitions were detected and analyzed for the peak-to-peak method, 598 transitions were detected and analyzed for the single threshold method, and 644 transitions were detected and analyzed for the change point algorithm. The same number of transitions were detected and analyzed for each method when estimating the binding termination times. Note that a negative average error indicates that the detected transitions occurred before the actual transitions, on average.

Table 3. The change point algorithm improves ensemble averages. 10 sets of data were simulated, each containing 100 binding interactions (sets 1-10). Interactions were detected using either the peak-to-peak or the single threshold method, and interactions 
941 were aligned using either the transitions estimated by the covariance threshold method

942 or the change points identified by the change point algorithm. For each data set, ensemble

943 averages were generated using either the known locations of actual simulated binding

944 interactions (real) or using the binding interactions detected by each method of analysis.

945 The averages were fit with exponential functions, and the substep sizes and rates of the

946 simulated myosin working stroke were estimated from the rates and amplitudes of the

947 exponential fits. (top) Mean and 95\% confidence intervals for the size of substep 1, the

948 size of substep 2, the total step size, and the rate of transitioning from the first substep to

949 the second substep, as estimated by the time forward ensemble averages. (bottom) Mean

950 and $95 \%$ confidence intervals for the size of substep 1 , the size of substep 2 , the total

951 step size, and the rate of transitioning from the second substep to the detached state, as

952 estimated by the time reversed ensemble averages. The $p$-value for a given set of

953 parameter values estimated by a given analysis method was obtained from the Wilcoxon

954 rank sum test between those estimated parameter values and the values estimated by

955 using the known locations of actual simulated binding interactions (real). 


\section{$956 \quad$ Figure 1}

A

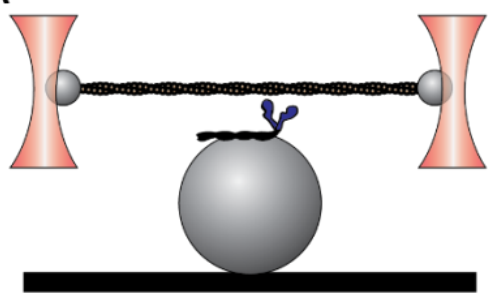

C

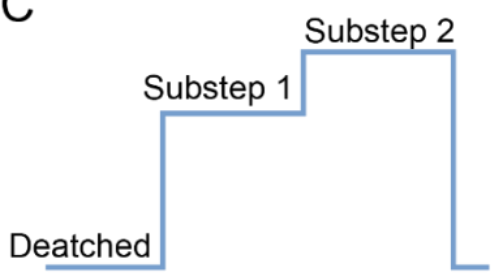

B
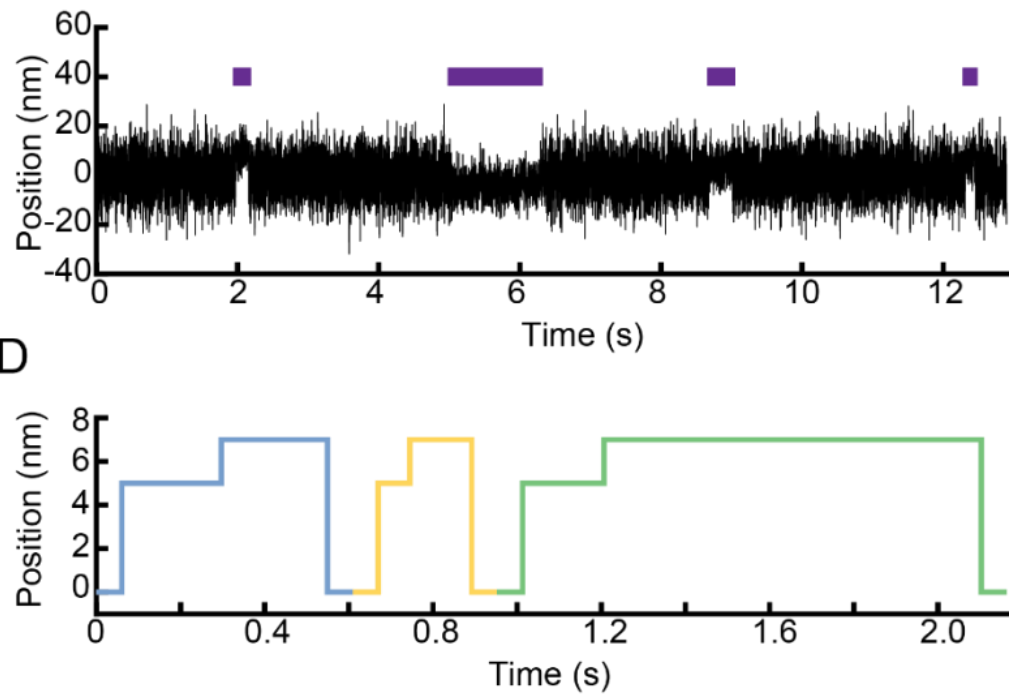

E
$\mathrm{F}$

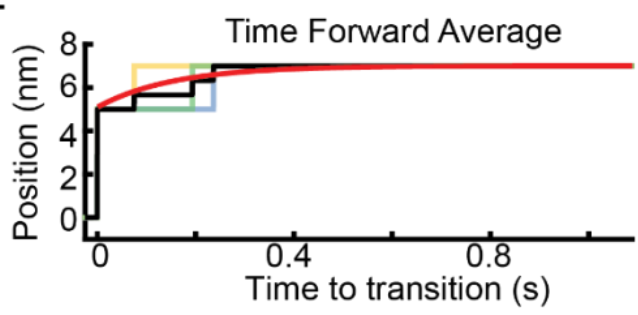

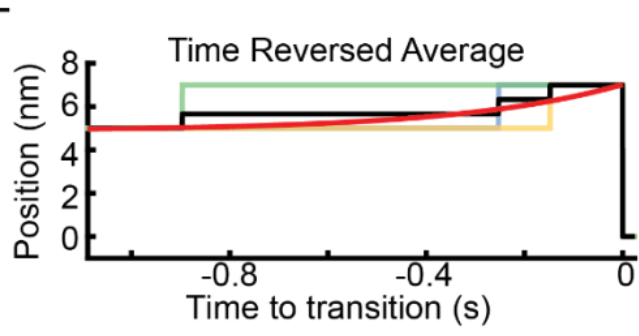




\section{$958 \quad$ Figure 2}

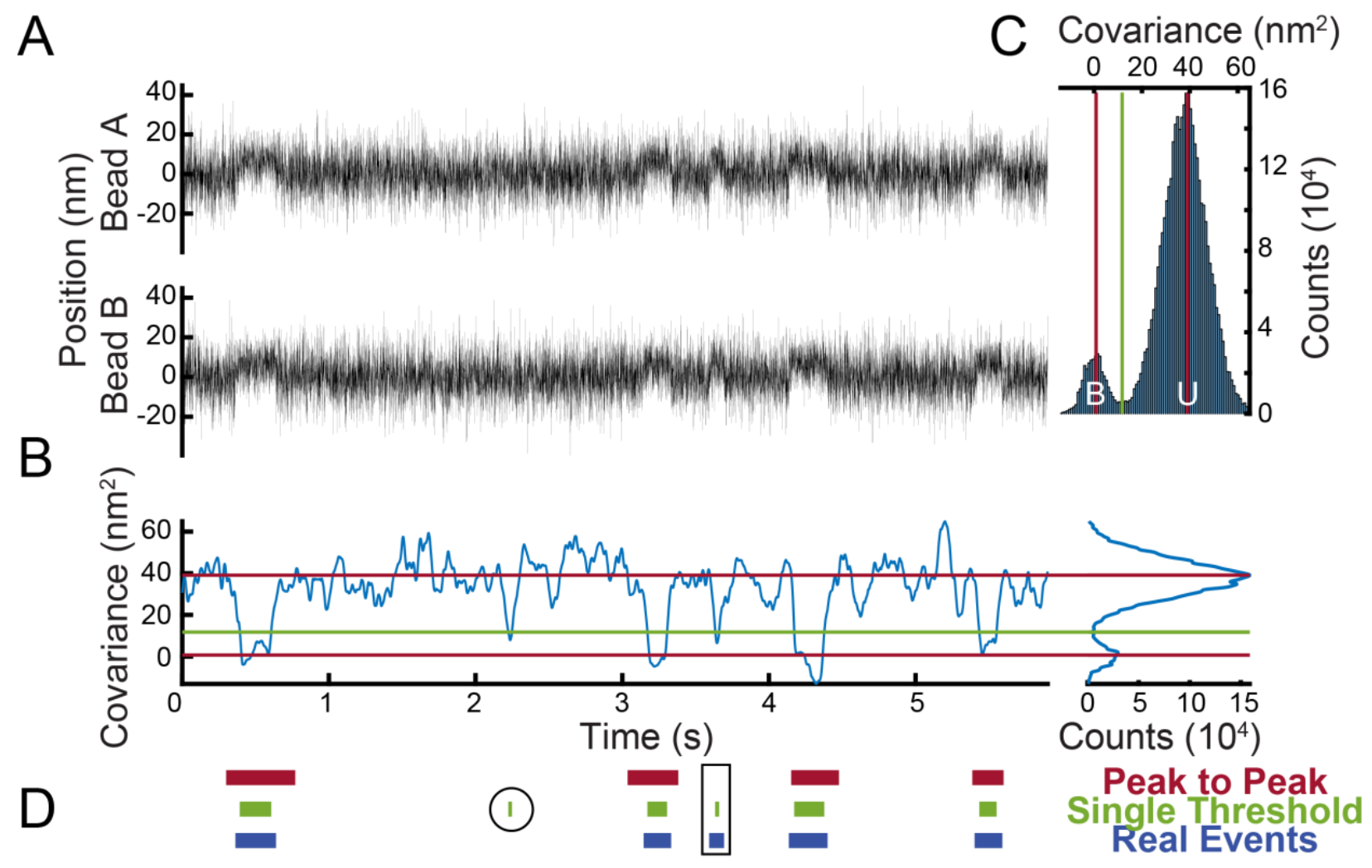


Figure 3

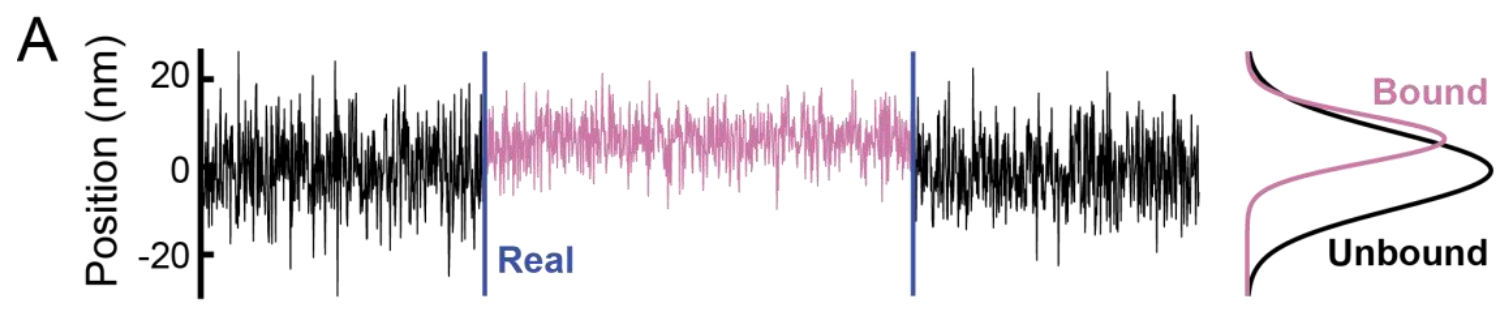

$\mathrm{B}$

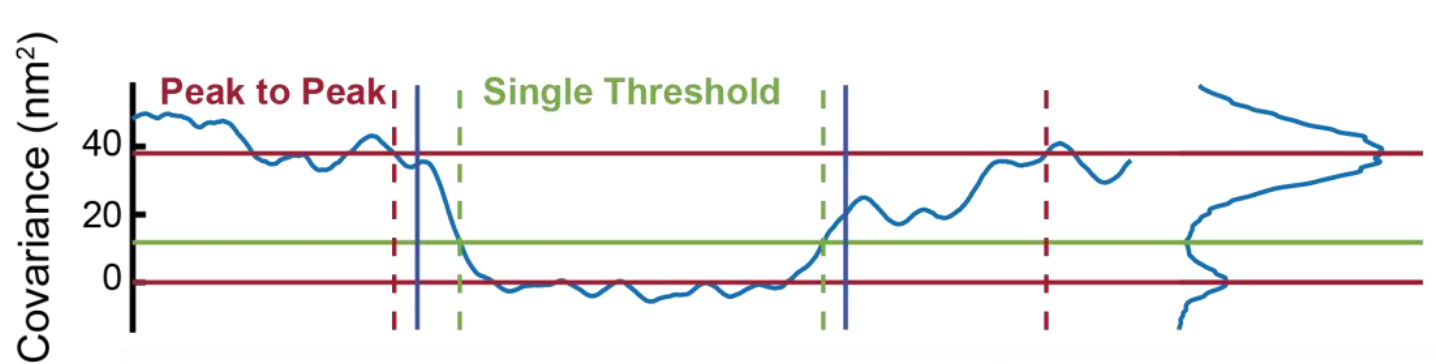

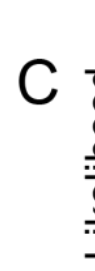

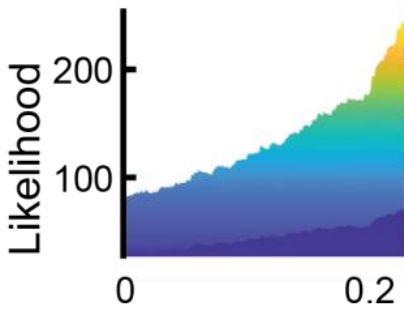

0.2
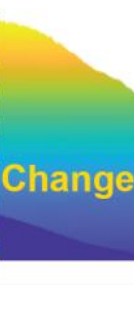

Time (s)

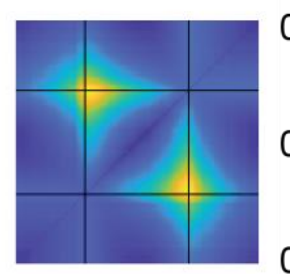

0.8 (5) 0.4 ह 


\section{$962 \quad$ Figure 4}

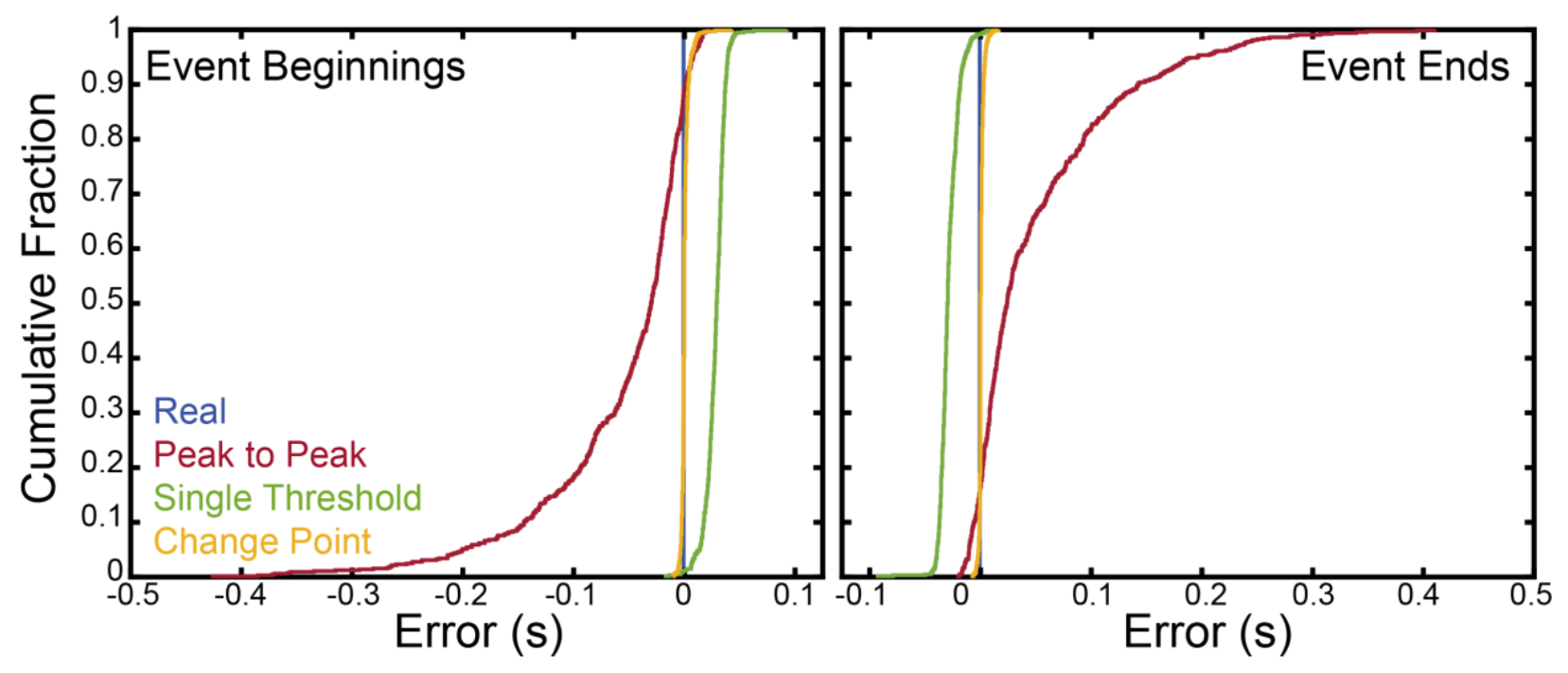


Figure 5
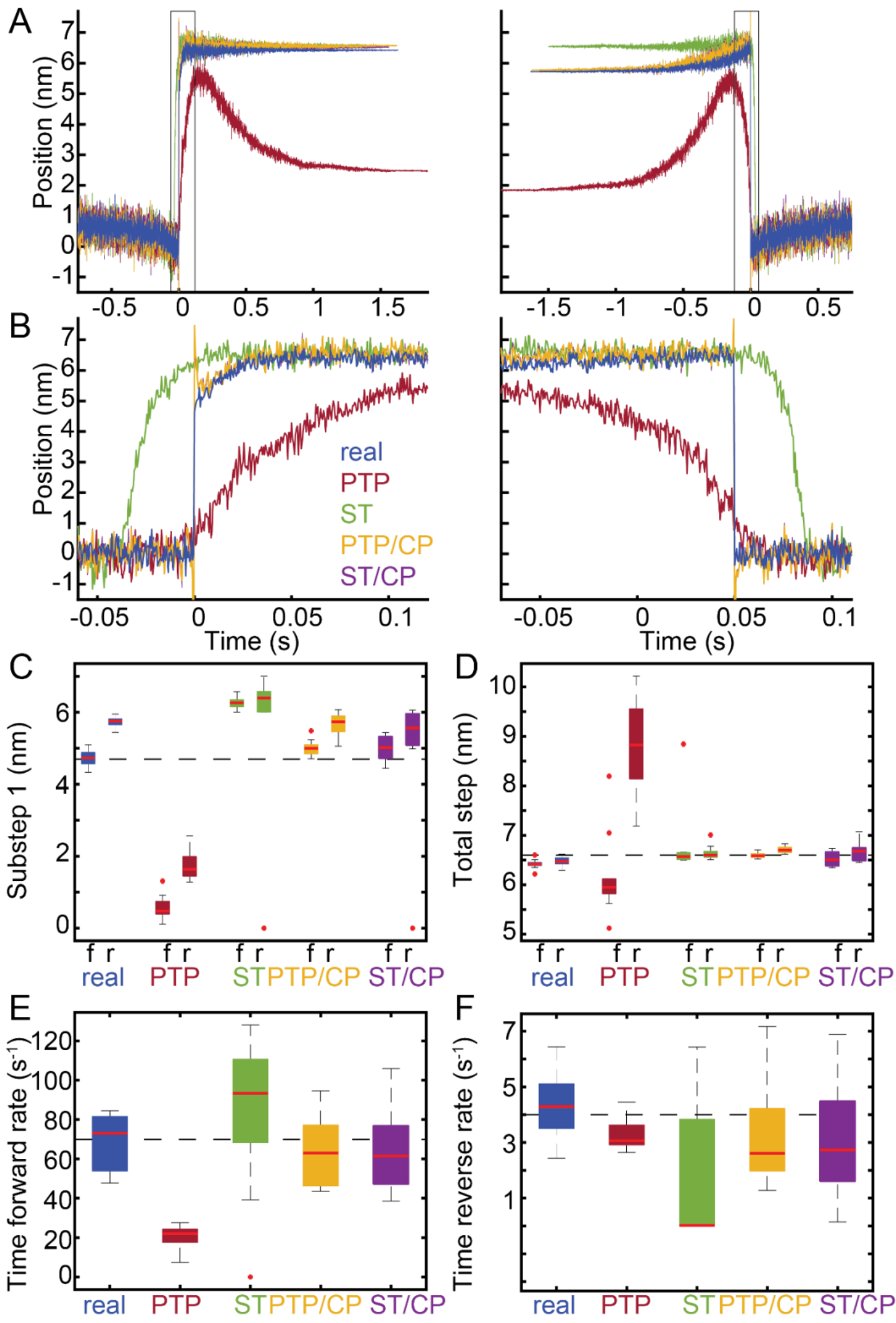
Figure 6

A

$\mathrm{D}(\mathrm{nm})$

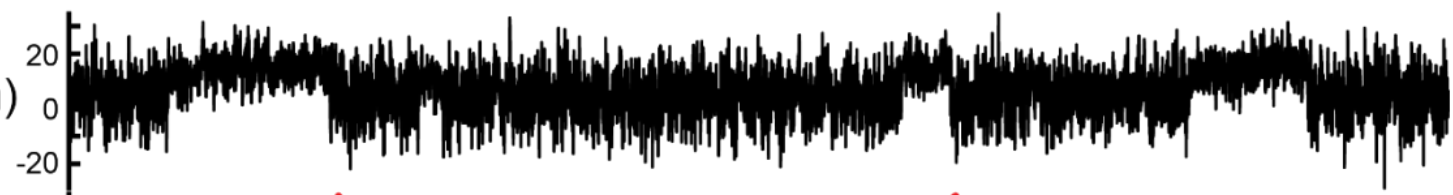

C $\left(\mathrm{nm}^{2}\right)$

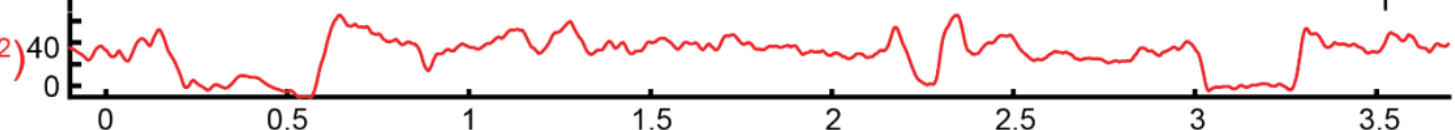

Time (s)

B
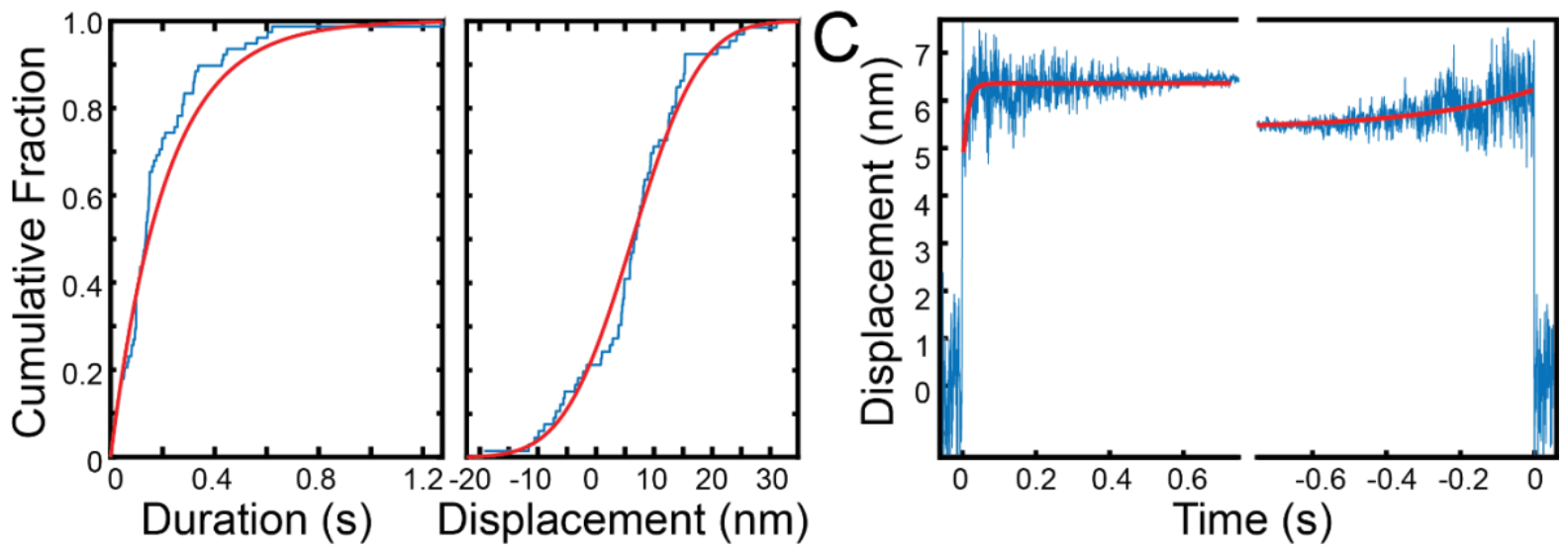


\section{Table 1}

969

\begin{tabular}{|c|c|c|c|}
\hline Method & $\begin{array}{c}\text { Average \# of correctly } \\
\text { detected interactions } \\
\text { (mean } \pm \text { SD) }\end{array}$ & $\begin{array}{c}\text { Average \# of } \\
\text { missed interactions } \\
\text { (mean } \pm \text { SD) }\end{array}$ & $\begin{array}{c}\text { \# of false positive } \\
\text { interactions / 100 } \\
\text { seconds (mean } \pm \text { SD) }\end{array}$ \\
\hline Single threshold & $80 \pm 4$ & $20 \pm 4$ & $4 \pm 1$ \\
\hline Peak-to-peak & $65 \pm 5$ & $36 \pm 5$ & 0 \\
\hline
\end{tabular}

970 
971 Table 2

972

\begin{tabular}{|c|c|c|}
\hline Method & $\begin{array}{c}\text { Error in binding initiation } \\
\text { times (ms, mean with 95\% } \mathbf{~ C l})\end{array}$ & $\begin{array}{c}\text { Error in binding termination } \\
\text { times (ms, mean with 95\% Cl) }\end{array}$ \\
\hline Single threshold & $28.2(+13.8,-21.7)$ & $-28.6(+19.1,-11.9)$ \\
\hline Peak-to-peak & $-55.5(+69.0,-195.5)$ & $50.4(+188.1,-64.9)$ \\
\hline $\begin{array}{c}\text { Change point } \\
\text { algorithm }\end{array}$ & $0.5(+9.0,-5.5)$ & $0.7(+4.8,-4.2)$ \\
\hline
\end{tabular}

973 
Table 3

975

\begin{tabular}{|c|c|c|c|c|c|}
\hline \multicolumn{6}{|c|}{ Time forward ensemble averages (mean with 95\% Cl) } \\
\hline Parameter & real & PTP & ST & PTP, CP & ST, CP \\
\hline Substep 1 (nm) & $\begin{array}{c}4.7 \\
(+0.4,-0.4)\end{array}$ & $\begin{array}{c}0.6 \\
(+0.7,-0.5) \\
p<0.001\end{array}$ & $\begin{array}{c}6.3 \\
(+0.3,-0.3) \\
p<0.001\end{array}$ & $\begin{array}{c}5.0 \\
(+0.5,-0.3) \\
p=0.021\end{array}$ & $\begin{array}{c}5.0 \\
(+0.4,-0.6) \\
p=0.045\end{array}$ \\
\hline Substep 2 (nm) & $\begin{array}{c}1.7 \\
(+0.5,-0.4)\end{array}$ & $\begin{array}{c}5.6 \\
(+1.7,-0.8) \\
p<0.001\end{array}$ & $\begin{array}{c}0.5 \\
(+1.7,-0.4) \\
p=0.003\end{array}$ & $\begin{array}{c}1.6 \\
(+0.3,-0.4) \\
p=0.427\end{array}$ & $\begin{array}{c}1.5 \\
(+0.5,-0.6) \\
p=0.186\end{array}$ \\
\hline Total step (nm) & $\begin{array}{c}6.4 \\
(+0.2,-0.2)\end{array}$ & $\begin{array}{c}6.2 \\
(+2.0,-1.1) \\
p=0.026\end{array}$ & $\begin{array}{c}6.8 \\
(+2.1,-0.3) \\
p=0.002\end{array}$ & $\begin{array}{c}6.6 \\
(+0.1,-0.1) \\
p=0.001\end{array}$ & $\begin{array}{c}6.5 \\
(+0.2,-0.2) \\
p=0.186\end{array}$ \\
\hline Rate (s-1) & $\begin{array}{c}68.7 \\
(+15.8,-20.9)\end{array}$ & $\begin{array}{c}20.2 \\
(+7.4,-12.8) \\
p<0.001\end{array}$ & $\begin{array}{c}84.3 \\
(+43.8,-84.3) \\
p=0.141\end{array}$ & $\begin{array}{c}64.5 \\
(+30.0,-20.9) \\
p=0.473\end{array}$ & $\begin{array}{c}63.6 \\
(+42.3,-25.0) \\
p=0.241\end{array}$ \\
\hline \multicolumn{6}{|c|}{ Time reversed ensemble averages (mean with 95\% Cl) } \\
\hline Parameter & real & PTP & ST & PTP, CP & ST, CP \\
\hline Substep 1 (nm) & $\begin{array}{c}5.7 \\
(+0.2,-0.3)\end{array}$ & $\begin{array}{c}1.7 \\
(+0.8,-0.4) \\
p<0.001\end{array}$ & $\begin{array}{c}5.2 \\
(+1.8,-5.2) \\
p=0.026\end{array}$ & $\begin{array}{c}5.7 \\
(+0.4,-0.6) \\
p=0.970\end{array}$ & $\begin{array}{c}5.0 \\
(+1.0,-5.0) \\
p=0.385\end{array}$ \\
\hline Substep 2 (nm) & $\begin{array}{c}0.7 \\
(+0.2,-0.2)\end{array}$ & $\begin{array}{c}7.1 \\
(+1.7,-1.4) \\
p<0.001\end{array}$ & $\begin{array}{c}1.4 \\
(+5.2,-1.4) \\
p=0.038\end{array}$ & $\begin{array}{c}1.0 \\
(+0.5,-0.4) \\
p=0.026\end{array}$ & $\begin{array}{c}1.6 \\
(+4.8,-1.1) \\
p=0.045\end{array}$ \\
\hline Total step (nm) & $\begin{array}{c}6.5 \\
(+0.1,-0.2)\end{array}$ & $\begin{array}{c}8.8 \\
(+1.4,-1.6) \\
p<0.001\end{array}$ & $\begin{array}{c}6.6 \\
(+0.4,-0.1) \\
p=0.003\end{array}$ & $\begin{array}{c}6.7 \\
(+0.1,-0.1) \\
p<0.001\end{array}$ & $\begin{array}{c}6.7 \\
(+0.4,-0.2) \\
p=0.011\end{array}$ \\
\hline Rate (s-1) & $\begin{array}{c}4.3 \\
(+2.2,-1.9)\end{array}$ & $\begin{array}{c}3.3 \\
(+1.2,-0.6) \\
p=0.054\end{array}$ & $\begin{array}{c}1.7 \\
(+4.8-1.7) \\
p=0.038\end{array}$ & $\begin{array}{c}3.3 \\
(+3.9,-2.0) \\
p=0.089\end{array}$ & $\begin{array}{c}3.0 \\
(+3.8,-2.9) \\
p=0.076\end{array}$ \\
\hline
\end{tabular}

